\title{
On Benchmarking of Invoice Analysis Systems
}

\author{
Bertin Klein, Stefan Agne, and Andreas Dengel \\ DFKI GmbH, 67663 Kaiserslautern, Germany \\ $\{k l e i n$, agne, dengel\}@dfki.de
}

\begin{abstract}
An approach is presented to guide the benchmarking of invoice analysis systems, a specific, applied subclass of document analysis systems. The state of the art of benchmarking of document analysis systems is presented, based on the processing levels: Document Page Segmentation, Text Recognition, Document Classification, and Information Extraction. The restriction to invoices enables and requires a more purposeful, i.e. detailed, targetting of the benchmarking procedures (acquisition of ground truth data, system runs, comparison of data, condensation into meaningful numbers). Therefore the processing of invoices is dissected. The involved data structures are elicited and presented. These are provided, being the building blocks of the actual benchmarking of invoice analysis systems.
\end{abstract}

\section{Introduction}

An interesting and important breed of document analysis systems is the specific class of systems that is devoted to the analysis of invoices: invoice analysis systems, IAS. There is not only a market for IAS, so that they are economically interesting $\mathrm{DNW}^{+} 03, \mathrm{KD}$ 04b. Also, they are a useful field to better understand details of the nature of document analysis systems under slightly restricted and thus slightly easier conditions. Lastly it is simply a good chance for a scientific field to potentially provide companies with research results and be elegantly able to prove their applicability KD04b.

With the advent of IAS, there emerges the need for a benchmarking of IAS. In order, either to assure the correctness of results, or to get clues about required corrections of the results and/or the IAS, it is crucial to (be able to) measure the quality of the results of IASes. The respective scientific discipline and the real, practical activity are called benchmarking.

It is without much doubt, that the following four analysis levels are core constituents of any useful analysis of documents in general and of invoices in particular:

- Document Page Segmentation

- Text Recognition

- Document Classification

- Information Extraction 
It is easy to imagine that if one level produces significantly bad results, then the following levels, depending on these bad results as input, will usually not be able deliver good results. Trying to hunt down the origin of some bad results, one would check the results after each of these four major levels. A benchmark of the complete process, thus needs to comprise benchmarking of the levels enumerated above.

Benchmarking, based on these levels, is well researched, i.e. measures have been developed, to calculate and denote representations of respective qualities. An overview is given in section 2. In order to benchmark an IAS, it is not sufficient to know these levels, their measures and their applicability. A deeper understanding of the practical application is required, i.e. what is been done with invoices, which steps constitute the processing of the invoice. An approach to invoice processing steps is made in section 3. The processing steps with an invoice imply the information fields that are needed from the invoice. The invoice processing practice surely judges IAS by their ablility to provide the required fields with high quality - even if there existed other IAS which were better in some other specific analysis task. Thus, these information fields are the hooks on which to benchmark an IAS. Only after having entered into this mechanism, one can substantiate the specification of ground truth requirements for the respective levels and start the collection and preparation of ground truth data. Finally, the concrete measuring activity can access the measures already introduced in section 2 .

\section{State of the Art}

This section provides an introduction and overview of benchmarking of document analysis systems (of which IAS are a subclass), along the four processing levels of: Document Page Segmentation, Text Recognition, Document Classification, and Information Extraction.

\subsection{Document Page Segmentation}

Two main classes of approaches for the benchmarking of document page segmentation can be distinguished in the literature: bitmap based approaches that operate at pixel level on the document bitmap and text based approaches that benchmark the segmentation at character level.

Bitmap Based Benchmarking: Randriamasy and Vincent are the names behind the origin of bitmap based evaluation [RV94a, RV94b, RVW94, YV95]. Their work has more recently been revisited, e.g. by $\left.\mathrm{PCL}^{+} 01\right]$ and more prominently e.g by Bre02. The document image (e.g. in TIFF format) is used and the zoning ground truth in which the regions are described by polygons. Also the result of the automatic zoning is needed in this format. The evaluation performs a geometrical comparison between the segmentation results and the zoning ground truth by testing the affiliation of each black pixel to corresponding regions. The 
quality of the segmentation is determined by the number of pixels or characters in the wrongly segmented regions of the document. Thulke et al. [TMD98] classify the errors into 19 different types.

Text Based Benchmarking: Text based evaluation operates on the text output of an OCR system. First the OCR system is applied only to the document image. The resulting output contains segmentation errors and OCR errors. Then the OCR system processes the same document image again, additionally provided with the manually generated zoning ground truth. The resulting text output of the second run contains only OCR errors. For both texts the error correction costs are computed by string matching algorithms (e.g. based on the Levenshtein edit distance). The difference then denotes the costs of correcting the segmentation errors KRN93, KRNN95.

Being solely text based, SEE ARR00, ADK03 combines the advantages of bitmap based and traditional text based evaluation systems. Contrary to the bitmap based approaches, SEE is able to evaluate the segmentation of OCR systems which do not provide the results of automatic zoning. Furthermore, the segmentation errors can be classified, which was not possible with the text based evaluation methods. The fact that SEE does not need the manually generated zoning ground truth as input leads to a reduction of effort and cost. As a side effect of this SEE can only approximate the number of true occuring segmentation errors.

\subsection{Text Recognition}

Text Recognition is the most elaborated field within document analysis with respect to benchmarking, mainly attributable to the extensive work at ISRI. The ISRI tools are a very good basis for the benchmarking of standard text recognition systems [RKN93, RKN94, RJN95, RJN96]. The most important measure at benchmarking the text recognition is the Character Accuracy. Further character based evaluation measure are: Confident Interval, Failure, Throughput, Marked Character Efficiency, Confident Metric, and Accuracy by Character Class. For more measures the characters are not treated isolatedly, but text is considered to consist of words separated by whitespace. The most prominent word-based measures are: Word Accuracy, Stopword Accuracy, Non-Stopword Accuracy, Distinct Non-Stopword Accuracy, Phrase Accuracy, and Accuracy by Word Length. Moreover, the effect of the class of input data has been considered, leading to: Page Quality Groups, Fax - Non-Fax, Effect of Resolution, Grayscale - Binary Image, Effect of Font Features, and Effect of Skew. Related research was done at the University of Washington, which however was more focused on error models and the creation of synthetic data Bai93, HB93, Bai95, HB95].

\subsection{Document Classification}

Most measures of document classification benchmarking base on the model of the contingency table, used to simply display the number of correctly and incorrectly classified documents. 
Table 1. Binary contingency table after Lew91, Lew95]

\begin{tabular}{|r|c|c|}
\hline & \multicolumn{2}{|c|}{ Ground Truth } \\
\hline System & $K$ & $\neg K$ \\
\hline \hline$K$ & $\mathrm{a}$ & $\mathrm{b}$ \\
\hline$\neg K$ & $\mathrm{c}$ & $\mathrm{d}$ \\
\hline
\end{tabular}

In the contigency table, $a$ denotes the number of documents, which have been correctly assigned to the class $K, b$ denotes the number of documents, which have been wrongly assigned to class $K$; wrong, because they are not documents belonging to that class. Most widely used are Precision and Recall [Lew91, Lew95], and the F-Measure [vR79, Lew95], which is calculated on top of both, precision and recall. Further, there are Reject Rate, Fallout, Error Rate, and Accuracy. For an accumulated measure over all classes Macro Averaging and Micro Averaging are used vR79, Lew91. There are also approaches, which consider costs of misclassifications, Cost Measure WK91.

\subsection{Information Extraction}

The basis to benchmark systems at information extraction are usually so-called Templates, patterns supposed to be filled by the system. A template consists of several Slots, in which information is entered in form of phrases. A template for the official report of a soccer game could have the slots: hometeam, guest, playground and result. The contents of a slot, provided as ground truth by a human is called Key, the value found by the system is called Response. The comparison of both leads to a distinction of six cases Chi92,CS93:

Correct: Response $=$ Key

Partia!1: Response $\cong$ Key

Incorrect: Response $\neq$ Key

Spurious: Key empty, Response not empty

Missing: Response empty, Key not empty

Noncommital: Key and Response are both empty

Basing on the above Chi92,CS93, LS91, define the following measures: Error, Undergeneration, Overgeneration, Substitution, Recall, Precision, F-Measure, and Error Rate per Word.

\section{Invoice Processing Step Ontology}

In oder to benchmark IASs, supposed to support companies or private persons in their handling of invoices, it is required to develop an understanding, what people do on the reception of an invoice, how the triggered process looks like, and what thus is important for companies or private persons to get from an IAS. Let somebody get the invoice in Figure 1 delivered onto his desk, i.e. either an

\footnotetext{
${ }^{1}$ At Muc-6[MUC95] Partial was not used any more, i.e. PAR was set to 0 for all tests.
} 
agent in a medical insurance company 2 , or a private person. What is required to do? We elicited the following differentiation into eleven different steps (not all of them mandatory). Note, that these steps represent some serious ontological committment already (which is why this section is entitled alike).

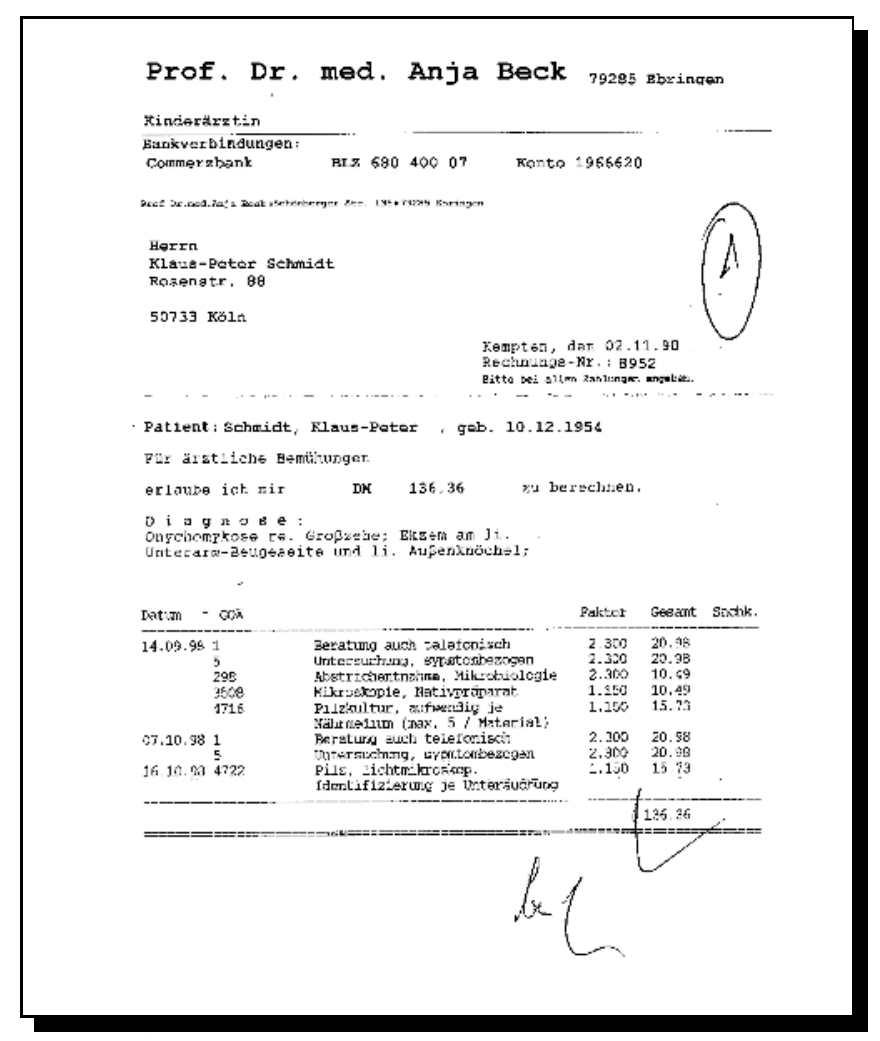

Fig. 1. A typical (in this case medical) invoice

This version elaborates on the version published in $\left[\mathrm{DNW}^{+} 03\right.$ :

1. Classification. Determining the class "invoice" triggers the processing steps decribed here. Further subclassing often makes sense.

2. Retrieval of contract. The patient needs to be spotted. Consider: addressee, firstname, lastname, birth date. A contract holder, or contract number could be specified, or a relation of patient to contract holder be mentioned ("son").

3. Retrieval of operation. Is there a folder with previous documents. Find the treatments and their dates in the invoice, the diagnosis. Conclude possible histories. Compare the (recent or timely related) activities which are logged with the contract (for the patient at hand).

$\overline{2}$ Taking a medical invoice as a model does not imply a loss of generality. 
4. Justification of contract plausibility. Do invoice address and contract address match? Are treatments admitted treatments, and took place while contract existed?

5. Decide payment target. Compensate the patient (if he already paid the doctor), the doctor or the doctors invoicing agency. What is: default, general rule in the company, usually better? Does the invoice have a due date, or other time constraints (so that the default rule is modified)?

6. Collect payment data. Spot account and bank (name and code of both), a reference number, a payment due date (permitting a delayed payment), comments (perhaps treatments or patient name or dates). Possibly extra comments are required, to explain reductions, etc. ...?

7. Justification of payment data plausibility. Is the location of the bank related to the recipient address? Do account name and code match with the data from the contract or possibly a company database of doctors. Does the reference number look genuine (i.e. "2313-AMB-12-1998" could raise doubts, because 1998 looks like a reference to the long ago year 1998)?

8. Justification of invoice correctness. Many invoices are not correct! Are all required information fields there, for invoices in general (like adressee, "trading good", tax number of invoicing party etc.), and for medical invoices (25\% reduction for hospital treatments, ICD diagnosis code, ...). Are calculations correct? Are balances carried over correctly? Are treatment codes correct and combinations allowed?

9. Justification of invoice plausibility. Do treatments coincide and suit the diagnosis? Is the sequence of treatments plausible?

10. Pay. Retrieve the amount (claimed amount minus possibly reductions). Fill out bank name and code, account name and code. Provide the invoice-nr and other required reference in the comment field. Provide further comments. Set the due date. Commit.

11. Archive and log. Write a line in the contract log. Open or continue an/the operation. Store the invoice. Store the data read. Document the payment. Document and explain any non-standard actions.

It could be problematic, if there occur unusual, special directives on invoices. In practice, it is not problematic. We did not know this from the beginning, but learned it only after a while of practically processing many invoices and large sums KDF04. Meanwhile some companies let our system process many invoices without any individual human control. Systems do not need to be perfect for that, it is enough when they commits less - especially less expensive - errors than human agents, which is by the way a reconfirmation that benchmarking, the assessment of errors, should be taken seriously.

This description of an invoice process is a solid ground to start detailing benchmarking of IAS.

\section{Information Fields Needed in the Processing Steps}

Essentially, what we have provided so far, is sufficient to design a good concept for benchmarking IAS. All the main activities of processing invoices have been 
presented 3 It should be clearer now, what is required from an IAS, and how benchmarking could approach IAS. For example it is now clearly imaginable that e.g. sum total is a sensitive information field and any benchmarking would surely closely evaluate the respective system results. Further, benchmarking also wants to distinguish the good from the even better, and a very good system, can outperfrom other good systems, if it was equally good in the area of sensitive data, but also especially good in some speciality e.g. in detecting seemingly minor inconsistencies in the justification tasks.

Although, the different concepts appearing in the processing descriptions might seem a confusing cocktail of very different kinds of concepts. By pinning down what they actually imply for a system, e.g. what values the concepts may take, the confusion starts to appear much easier to master. This is done now. 4

\subsection{Data-Oriented Summary of the Process Steps}

With some revisiting and re-ordering, first, the process steps can be clustered together, as they are not so different from each other like they first seem. Second, the quality of the link between input data and output data can be characterized (in the following section). Independent of the algorithm inside some IAS, it is then (and only then) possible to prepare ground truth data for a domain and then benchmark its behavior.

The finally needed information fields are: "payment data", i.e. "payment target", "bank (name and code)", "bank account (name and code)", "reference number". Prior to that, for the justification steps, some more information fields are necessary. Elements, or intermediate results of justification are single notes of "plausibility", e.g. "plausibility of contract data", and their consequences: "acceptance of invoice", "correctness of invoice", "reduction (of claimed amount)", "explanation (of reduction)", "genuine look of a code". One speciality is "urgency", a distance relation on some dates.

All the justification is to a very large degree based on matching patterns, which read as "comparison of data (e.g. addresses)". To a much smaller degree it sometimes bases on a "proximity (or relation) of locations" and also sometimes on the construction of an "interpretable story of dates" (or at least collection and display of dates to the user).

There exist some general processing schemes: "(default) business rule", "law", "general use". Further, note that between the above concepts, which are searched for, and the following concepts, which are those appearing in documents, there

${ }^{3}$ Actually, also the software engineers who coded our system KD04a started their work with descriptions on such a level. Some people even say, and we partly adhered to that, that a benchmarking concept should be constructed prior to the implementation of a system in order to assure a system meet its requirements well.

${ }^{4}$ Individual mileages may vary. The way we proceed is not unique. It is very usual to encounter differences, when talking about conceptualizations. However, with varying conceptions, as long as they are only consistently used, one can still reach the same result $\mathrm{KBC}^{+} 05$. 
are often some bridging concepts. E.g. addressee is part of an address, but can also become a patient or contract holder and a payment target.

\subsection{Information Field Concept Classes}

The whole set of information fields appearing on invoices can be distinguished into the following set of classes.

1. Enumerable concepts: A number of concepts appearing on invoices can be judged as correctly recognized or not, if one only has a databast 5 in which all their different possible values are stored, like: "country:= New Zeeland, Italy, Germany, Great Britain, USA,...". We consider the following concepts to be of that kind: treatment, a patient (respectively a patient identification, usually firstname, lastname, date-of-birth), a diagnosis, location of bank, a doctors address, treatment code, allowed treatment combination 6

2. Record concepts: There is another kind of database either available in companies anyhow, or possible to construct. They can be used in the first place to map facts to other facts. The database with contracts is the main such database. From there one can retrieve all "contracts" and get their "contract data", i.e. contract number, contract holder, operation, contract duration, contract address, contract coverage (which treatments).

Other databases could cover "treatment suitabilities for diagnoses", and "possible histories (from treatment to diagnosis to cause)." To extend an invoice system for classes other than invoices, requires a database with the document classes linking to specifications of their required processing.

3. Visual databases: Depending on the details of a domain, and given that there is a set of visual matching operations, which are extremely quick, it is in a number of cases found that it is worked with databases of 2 dimensional layout templates (reduced/abstracted representations of real layouts), to map between pages and classes or page snippets and ROI classes (e.g. Figure or Table). (This addresses the implications of the widespread strategy to exploit that many recipients receive many invoices from one sender, who often use fixed basic templates for their invoices, e.g. built-in in their invoicing software [KGKD01].

4. Labelled concepts: These are the concepts which are (most often) simply identified, because a keyword indicates what comes next, "From: ....". These concepts can also be treated with a database alike the one for enumerable concepts, but the database has to store the keywords or labels (e.g. "From", "Total", "Sum", "Amount", "Euro", "Diagnosis",...). Concepts of this category are: claimed amount, the patient, the diagnosis.

${ }^{5}$ Please read "ontology" instead of "database" from now on, if you know how to store knowledge in ontologies.

${ }^{6}$ In our system, we frequently use this even to actually spot -i.e. not benchmark but spot- occurences of peoples names, including permutations, spelling errors, and OCR errors, which is still very quick with databases of 1 Million names [KDF04]. 
5. Syntactic concepts: This simply bundles all information fields which are convenient to describe with something like a regular expression, or a similar means of abbreviated description. Typical for this category are all kinds of dates: letter date, date of a treatment, payment due date, but also "a calculation (not doing one, but something printed like: "1 $+2=3$ "!). Also addresses are often described syntactically: address, the invoice address, addressee, doctors address. In early applications we described tables of treatments also syntactically (mainly because they often exhibit well- behaved columns with numbers).

We subsume another group of concepts here, those characterized by their layout. The only occurrences in our example invoice are: subject and reference-field. However, the reference-field can be determined also with a clear reference to its structure. Only the benchmarking of the subject spotting feature needs to be purely based on its look, position, and bold font.

6. Secondary concepts: To finish, one more recognition feature to be benchmarked has to be mentioned (c.f. Sum98]). In general, there exist concepts of elements visible on documents, (called secondary in Sum98]), which might be identifiable only after other elements have been readily identified. To use the prior, intermediate search results, is a valuable and simple strategy anyhow. If the address was found, no other adress has to be searched, as well as everything belonging to the address, needs not be considered in further searches (for subject, diagnosis, ...). Here, the last concepts come into play, that are simple to find out and represent in the system: "the required information fields for (German) invoices in general", and "required information fields for (German) private medical invoices". Now, for a specific application or class of IAS a benchmarking concept can be constructed based on these building blocks.

\section{Conclusion}

Good recognition of numbers is obviously very important when dealing with documents about money and its transfer. However, we hope to have contributed to some more understanding of requirements of IAS and dependencies on possibly crucial qualities and features, i.e. on IAS benchmarking.

When we conceived of this paper for the first time a couple of years ago, we wanted to call it something like: "the misunderstanding that there exists something like one ground truth". There is a cornucopia of work pointing out the serious problems, often in conjunction with high costs, when something is too quickly labelled correct or true, and slightly different viewpoints of important stakeholders cause these stakeholders to disagree. However, we have learned from practice that our approach must be more constructive, i.e. we need to try to show ways instead of showing the dead ends. Users or customers dont know what is helpful in the end, and worth their money, but they find it out later, because then the system they chose either makes their life better or not. It is important to assure the users success. Thus, we developed the presented checklist of scenario pieces, which enabled us to (conveniently) assure this in all the scenarios we encountered so far. 
We have presented the state of the art of basic benchmarking measures available. Then we have sketched the process, that is initiated when a private person or company receives an invoice. We have derived from this sketch simpler subtasks and the data required for them, which we have grouped into six classes. The sketch of the process should be used for practical projects to assign individual importances to the subtasks and the involved information requirements. These will then inform the choice of state of the art measures to use (E.g. we chose different measures for domains with invoices only below 100 Euro, than for domains with invoices above 100000 Euro, because the importance of the sum total field was specified differently). The classes of data, are the basis to guide the collection of ground truth data (for one customer and his specific "viewpoint") and can also serve to guide their application by the benchmarking system (as the classes tell something about the datatypes).

Future work is devoted to a closer inclusion of tables.

\section{References}

[ADK03] Stefan Agne, Andreas Dengel, and Bertin Klein. Evaluating see - a benchmarking systems for document page segmentation. In Proceedings of the 7th International Conference on Document Analysis and Recognition ICDAR'03), volume I, pages 634 - 638, Edinburgh, Scotland, United Kingdom, August, 3-6 2003.

[ARR00] Stefan Agne, Markus Rogger, and Jörg Rohrschneider. Benchmarking of document page segmentation. In Daniel P. Lopresti and Jiangying Zhou, editors, Document and Recognition and Retrieval VII, volume 3967 of Proceedings of SPIE, pages 165 - 171, San Jose, California, USA, 2000.

[Bai93] Henry S. Baird. Document image defect models and their uses. In Proceedings of the Second International Conference on Document Analysis and Recognition ICDAR, pages 62-67, Tsukuba Science City, Japan, October 20-22 1993. IEEE Computer Society Press.

[Bai95] Henry S. Baird. Document image defect models. In Lawrence O'Gorman and Rangachar Kasturi, editors, Document Image Analysis, pages 315325. IEEE Computer Society Press, 1995.

[Bre02] T. M. Breuel. Representations and metrics for off-line handwriting segmentation. In 8th International Workshop on Frontiers in Handwriting Recognition, 2002.

[Chi92] Nancy Chinchor. MUC-4 evaluation metrics. In Proceedings of the Fourth Message Understanding Conference (MUC-4), pages 22-29, McLean, Virginia, USA, June 16-18 1992. Morgan Kaufmann Publishers, Inc.

[CS93] Nancy Chinchor and Beth Sundheim. MUC-5 evaluation metrics. In Proceedings of the Fifth Message Understanding Conference (MUC-5), pages 69-78, Baltimore, Maryland, USA, August 25-27 1993. Morgan Kaufmann Publishers, Inc.

$\left[\mathrm{DNW}^{+} 03\right]$ A. Dengel, P. Nowak, C. Wagner, K. Rehders, B. Klein, D. Schneider, M. Winkler, and Tebel R. Studie automatisierte rechnungseingangsbearbeitung marktpotential, marktübersicht und trends. commercial study, September 2003. 
[HB93] Tin Kam Ho and Henry S. Baird. Perfect metrics. In Proceedings of the Second International Conference on Document Analysis and Recognition ICDAR, pages 593-597, Tsukuba Science City, Japan, October 20-22 1993. IEEE Computer Society Press.

[HB95] Tin Kam Ho and Henry S. Baird. Evaluation of OCR accuracy using synthetic data. In Proceedings of the Fourth Annual Symposium on Document Analysis and Information Retrieval SDAIR 95, pages 413-422, Las Vegas, Nevada, April 24-26 1995.

$\left[\mathrm{KBC}^{+} 05\right] \quad$ A. Kumar, A. Burgun, W. Ceusters, J. Cimino, J. Davis, P. Elkin, I. Kalet, A. Rector, J. Rice, J. Rogers, S. Schulz, K. Spackman, D. Zaccagini, P. Zweigenbaum, and B. Smith. Six questions on the construction of ontologies in biomedicine. In AMIA, Washington DC, USA., 2005.

[KD04a] Bertin Klein and Andreas Dengel. Problem-adaptable document analysis and understanding for high-volume applications. International Journal on Document Analysis and Recognition, 2004.

[KD04b] Bertin Klein and Andreas Dengel. Results of a study on invoice-reading systems in germany. In IAPR International Workshop on Document Analysis Systems, 2004.

[KDF04] Bertin Klein, Andreas Dengel, and Andreas Fordan. Reading and Learning - Adaptive Content Recognition, volume 2956 of LNCS, chapter smartFIX: An Adaptive System for Document Analysis and Understanding, pages 166 - 186. Springer, 2004.

[KGKD01] B. Klein, S. Gökkus, T. Kieninger, and A. Dengel. Three approaches to industrial table spotting. In Int. Conf. On Document Analysis and Recognition (ICDAR) '01, 2001.

[KRN93] Junichi Kanai, Stephen V. Rice, and Thomas A. Nartker. A preliminary evaluation of automatic zoning. In Kevin O. Grover, editor, Annual Research Report, pages 35-45, University of Nevada, Las Vegas, 1993. Information Science Research Institute.

[KRNN95] Junichi Kanai, Stephen V. Rice, Thomas A. Nartker, and George Nagy. Automated evaluation of OCR zoning. IEEE Transactions on Pattern Analysis and Machine Intelligence, 17(1):86-90, January 1995.

[Lew91] David D. Lewis. Evaluating text categorization. In Proceedings of the Workshop on Speech and Natural Language, pages 312-318, Pacific Grove, California, USA, February 19-22 1991.

[Lew95] David D. Lewis. Evaluating and optimizing autonomous text classification systems. In Edward A. Fox, Peter Ingwersen, and Raya Fidel, editors, Proceedings of the Eighteenth Annual International ACM SIGIR Conference on Research and Development in Information Retrieval SIGIR '95, Special Issue of the SIGIR Forum, pages 246-254, Seattle, Washington, USA, July 9-13 1995. ACM Press.

[LS91] Wendy Lehnert and Beth Sundheim. A performance evaluation of textanalysis technologies. AI magazine, 12(3):81-94, Fall 1991.

[MUC95] Proceedings of the Sixth Message Understanding Conference (MUC-6), Columbia, Maryland, USA, November 6-8 1995. Morgan Kaufmann Publishers, Inc. Inhaltsverzeichnis.

$\left[\mathrm{PCL}^{+} 01\right] \quad$ Liangrui Peng, Ming Chen, Changsong Liu, Xiaoqing Ding, and Jirong Zheng. An automatic performance evaluation method for document page segmentation. In Proceedings of the Sixth International Conference on Document Analysis and Recognition (ICDAR), pages 134 - 137, Seattle, Washington, USA, September 10-13 2001. IEEE Computer Society Press. 
[RJN95] Stephen V. Rice, Frank R. Jenkins, and Thomas A. Nartker. The fourth annual test of OCR accuracy. In Andrew D. Bagdanov, editor, Annual Research Report, pages 11-49, University of Nevada, Las Vegas, 1995. Information Science Research Institute.

[RJN96] Stephen V. Rice, Frank R. Jenkins, and Thomas A. Nartker. The fifth annual test of OCR accuracy. Technical Report TR-96-01, Information Science Research Institute, University of Nevada, Las Vegas, USA, April 1996.

[RKN93] Stephen V. Rice, Junichi Kanai, and Thomas A. Nartker. An evaluation of OCR accuracy. In Kevin O. Grover, editor, Annual Research Report, pages 9-33, University of Nevada, Las Vegas, 1993. Information Science Research Institute.

[RKN94] Stephen V. Rice, Junichi Kanai, and Thomas A. Nartker. The third annual test of OCR accuracy. In Kevin O. Grover, editor, Annual Research Report, pages 11-38, University of Nevada, Las Vegas, 1994. Information Science Research Institute.

[RV94a] S. Randriamasy and L. Vincent. Benchmarking page segmentation algorithms. In Proceedings of the 1994 IEEE Computer Society Conference on Computer Vision and Pattern Recognition, pages 411-416, Seattle, Washington, USA, June 21-23 1994. IEEE Computer Society Press.

[RV94b] Sabine Randriamasy and Luc Vincent. A region-based system for the automatic evaluation of page segmentation algorithms. In Andreas Dengel and A. Lawrence Spitz, editors, Proceedings of the International Association for Pattern Recognition Workshop on Document Analysis Systems DAS94, pages 29-41, Kaiserslautern, Germany, October 18-20 1994.

[RVW94] Sabine Randriamasy, Luc Vincent, and Ben Wittner. An automatic benchmarking scheme for page segmentation. In Proceedings of the ISET/SPIE 1994 International Symposium on Electronic Imaging Science and Technology, volume 2181, pages 217-230, 1994.

[Sum98] K. Summers. Automatic Discovery Of Logical Document Structure. PhD thesis, Cornell University, 1998.

[TMD98] M. Thulke, V. Märgner, and A. Dengel. A general approach to quality evaluation of document segmentation results. In S.-W. Lee and Y. Nakano, editors, Proceedings of the International Association for Pattern Recognition Workshop on Document Analysis Systems DAS98, pages 79-88, Nagano, Japan, November 1998.

[vR79] C. J. van Rijsbergen. Information Retrieval, chapter 7 Evaluation, pages 144-183. Butterworths, second edition, 1979.

[WK91] Sholom M. Weiss and Casimir A. Kulikowski. Computer Systems That Learn, chapter 2 How to Estimate the True Performance of a Learning System, pages 17-49. Morgan Kaufmann Publishers, Inc., 1991.

[YV95] Berrin A. Yanikoglu and Luc Vincent. Ground-truthing and benchmarking document page segmentation. In Proceedings of the Third International Conference on Document Analysis and Recognition, volume 2, pages 601-604, Montréal, Canada, August 14-16 1995. IEEE Computer Society Press. 